\title{
Candida spp. in Lower Respiratory Tract Secretions - A Ten Years Retrospective Study
}

\author{
Cristina Nicoleta Ciurea1,2, Ario Santini ${ }^{3}$, Anca Delia Mare ${ }^{1 *}$, Irina Bianca Kosovski2,4, \\ Felicia Toma ${ }^{1}$, Camelia Vintila5, Ionela Anca Pintea-Simon ${ }^{1,2}$, Adrian Man ${ }^{1,5}$ \\ ${ }^{1}$ Department of Microbiology, George Emil Palade University of Medicine, Pharmacy, Science, and Technology of \\ Targu Mures, Romania \\ 2 Doctoral school, George Emil Palade University of Medicine, Pharmacy, Science, and Technology of Targu Mures, \\ Romania \\ 3 George Emil Palade University of Medicine, Pharmacy, Science, and Technology of Targu Mures, Romania \\ 4 Department of Pathophysiology, George Emil Palade University of Medicine, Pharmacy, Science, and Technology of \\ Targu Mures, Romania \\ 5 Mureș Clinical County Hospital, Targu Mures, Romania
}

\begin{abstract}
Introduction: Lower respiratory tract secretions (LRTS) like sputum and tracheal aspirates are frequently sent to the microbiology laboratory from patients with various respiratory pathologies. Improper collection techniques can lead to false-positive results, resulting in improper therapy. Aim of the study: To determine the percentage of contaminated samples sent to the microbiology laboratory, to establish the prevalence of Candida spp. in non-contaminated samples and therefore, the presence of Candida spp. originating in lower respiratory tract infections. Material and Methods: A 10-year data survey was conducted to assess the differences in Candida prevalence from contaminated versus non-contaminated samples, assessed and categorised by Bartlett grading system, and to emphasise the importance of quality control for potentially contaminated samples. The data were analysed according to gender, age, referring departments, and the species of Candida. For the statistical analysis, Kruskal-Wallis and Fisher tests were used, and the alpha value was set for 0.5. Results: The prevalence of Candida spp. in all analysed samples was $31.60 \%$. After excluding the contaminated samples, the actual prevalence was $27.66 \%$. Of all sputum samples, $31.6 \%$ were contaminated. Patients aged more than 40 years old were more prone to provide contaminated sputum samples. C. albicans is more prevalent in non-contaminated sputum samples. In both sputum and tracheal aspirates, the chances of identifying a single species are higher than the chances of identifying multiple species. Conclusions: The study emphasises the importance of assessing the quality of sputum samples because of the high number of improperly collected samples sent to the microbiology laboratory.
\end{abstract}

Keywords: Candida, lower respiratory tract infections, contamination, laboratory diagnosis

Received: 17 March 2021 / Accepted: 24 May 2021

\section{INTRODUCTION}

Candida spp. are frequently isolated from respiratory tract secretions, but, as they normally colonise the oral cavity, the microbiological examination's clinical importance is questionable. Because Candida spp. are opportunistic pathogens, infections occur mainly in immunocompromised hosts. The higher risk population includes patients admitted to Intensive Care Units (ICU) because they have an impaired immune system and are exposed to various medical devices and invasive manoeuvres. Collecting lower respiratory tract secretions (LRTS) for the microbiological examination is a widely used diagnostic tool that can help identify Candida spp. However, interpreting the value of such examinations proves to be challenging: the yeast cells can be harmless commensal inhabitants of the respiratory tract, or they can express increased pathogenicity, by disseminating to various host niches to cause infections. The case fatality ratio of invasive infections in ICU is $45.9 \%$, making Candida spp. infections a significant health problem [1].

Sample collection techniques have a high impact on the accuracy of a laboratory diagnosis. The collection 
of sputum, in particular, poses challenges for patients and medical workers, and often saliva mislabelled as sputum is sent to a laboratory. Using sputum quality indicators such as the Q score or Bartlett score, samples contaminated with saliva can be ruled out after the microscopic examination [2].

Nevertheless, if a lower respiratory tract infection (LRTI) is indeed present, a sample may be ruled out because of the supplementary contamination with saliva by following the Q score alone. It is well known that culturing saliva will not provide enough evidence for diagnosing LRTIs, but despite this, the number of contaminated samples sent to laboratories is still high. Endotracheal aspirate samples are more qualitative than sputum, but endotracheal tubes are colonised quickly by oropharynx bacteria and yeast cells.

The oral mycobiome composition is dynamically modified over time, and underlying diseases can lower the threshold for opportunistic infections. Candida spp. are notorious for being part of the oral mycobiome, and the colonisation state of the oral cavity is often associated with deteriorating general health, medications, and denture wearing [3]. The whole picture of the clinical implications of the Candida spp. colonisation is not entirely understood, although there is an association between oral colonisation and the onset of infections, especially in hospitalised elderly patients [4]. Patients with prolonged hospitalisation and risk factors for invasive infections might benefit from early and appropriate antifungal therapy [5]. The economic impact of Candida spp. colonisation in patients discharged alive from the hospital was an added 12908 euros/patient, while in Spain a Candida spp. infection brings an added 21075 euro/patients in costs [6]. In the USA, Candida spp. are ranked the fourth most common pathogen responsible for bloodstream infections and the third most common in the ICU [7]. The mortality rate of candidemia ranges from $28 \%$ in young patients to $45 \%$ in elderly patients [8]. Recently, Candida spp. associate increased mortality in internal medicine yards $[8,9]$, raising serious concerns about new risk factors and bringing into the light the necessity for diagnostic algorithms that can help select patients who would most benefit from preventive antifungal treatment.

With a reported global emergence of non-albicans species $[10,11]$, monitoring fungal infections is crucial, especially for ICU patients, as intubation and mechanical ventilation are risk factors for Candida spp. colonisation.
The study aimed to:

- Determine the percentage of contaminated samples sent to the microbiology laboratory.

- Establish the prevalence of Candida spp. in noncontaminated samples and therefore, the presence of Candida spp. originating in lower respiratory tract infections.

\section{MATERIALS AND MeTHODS}

From the complete data base of reports of the Microbiology Laboratory, Mures Clinical County Hospital, between Jan 1, 2009, to 31th of December 2018, all reports on respiratory tract samples, sent to and processed in the microbiology laboratory were scrutinised for Candida spp. growth. Of these, 3199 sputum and tracheal aspirates were selected for inclusion in the study. Incomplete reports were excluded.

The sputum samples were further scrutinised for the sample's quality and assessed using the Bartlett (Q) grading system. The presence of leukocytes, mucus and squamous epithelial cells was observed under the microscope in 10 low power fields (LPF) $(<10$ neutrophils/LPF $=0$; 10-20 neutrophils/LPF $=+1$; $>$ neutrophiles $/ \mathrm{LPF}=+2$; the presence of mucus $=+1 ; 10-25$ squamous epithelial cells/LPF $=-1 ;>25$ squamous epithelial cells/LPF $=-2$ ). A final score value of less than 1 indicates significant salivary contamination of sputum, and these samples were considered and designated as "contaminated". A final score of 1 and above was considered to indicate a "non-contaminated "sputum sample, with marks of inflammation.

The tracheal aspirates were considered non-contaminated products for microbiological analysis, due to the aseptic way of harvesting.

The non-contaminated sputum samples plus all tracheal aspirates showing Candida spp. were considered relevant for diagnosing lower respiratory tract infection associated with Candida spp. (Figure 1).

Candida spp. were identified by culturing on Sabouraud agar. The samples that showed fungal growth were then subcultured on selective chromogenic agar for species identification. In selected cases, an automated system of identification (VITEK 2C) was used.

The results were analysed considering gender, age, referring departments, Candida species (C. albicans, $C$. non-albicans, and C. albicans + C. non-albicans). 


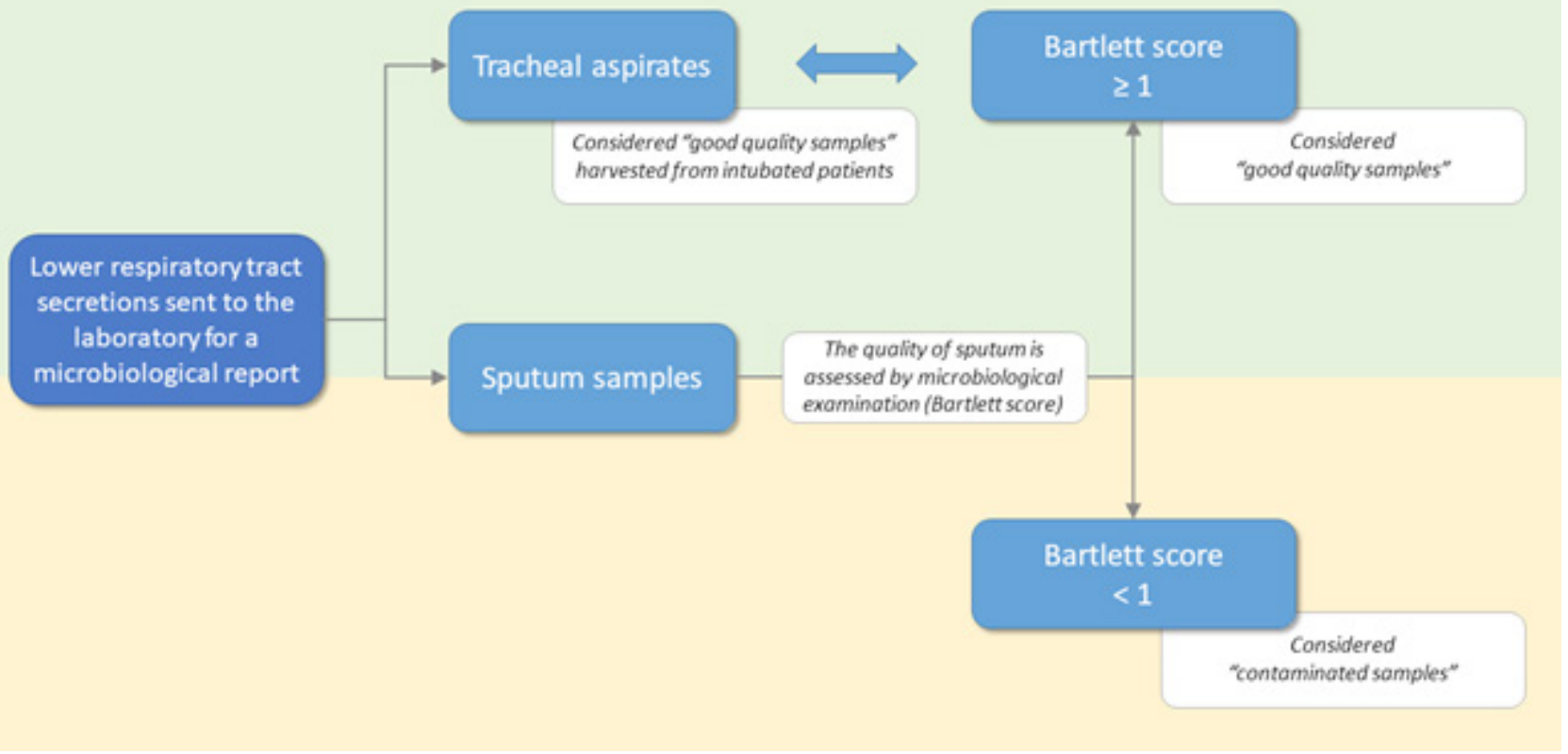

Fig. 1. Flowchart of processed samples

Patients were categorised into the different risk groups according to the wards to which they had been admitted (Oncology, Critical Care, medical wards Gastroenterology, Cardiology, Nephrology, Internal Medicine, Infectious Diseases), and surgical wards General Surgery, Plastic Surgery).

The obtained data were analysed using the KruskalWallis test to compare mean values and the Fisher test for nominal data. The alpha value was set for 0.05 .

\section{RESULTS}

The percentage of contaminated sputum samples testing positive for Candida spp. was 31.60\% (164 out of 519 analysed sputum samples). Of the total of lower respiratory tract samples (sputum plus tracheal aspirates), the prevalence was $35.76 \%$ (1144 of 3199 samples). After excluding the contaminated samples, the recalculated prevalence of Candida spp. in lower respiratory tract samples was $27.66 \%$.

The prevalence in all lower respiratory tract samples ranged from a minimum of $26.94 \%$ in 2012 to a maximum of $43.45 \%$ in 2011 (Figure 2).

The total number of samples tested positive for Candida spp., 613 (53.58\%) were sputum samples, and 531 $(46.42 \%)$ were tracheal aspirates. Based on the quality of sputum samples, $164(31.6 \%)$ were assigned a Bart- lett score $<1$ and were excluded from further analysis, while $355(68.40 \%)$ were assigned a Bartlett score $\geq 1$ and considered non-contaminated.

From the total number of samples:

- 607 (53.06\%) were collected: $531(86.72 \%)$ tracheal aspirates and 76 (13.10\%) sputum samples;

- 366 (31.99\%) sputum were collected from patients admitted to medical wards;

- 114 (9.97\%) sputum were collected from oncologic patients;

- 39 (3.41\%) sputum were collected from surgical wards;

- For 18 samples (1.57\%), the location could not be established

In LRTS considered to be non-contaminated, a statistically significant difference was observed between the average age of the patients that showed colonisation with C. albicans and C. non-albicans in LRTS (KruskalWallis Test; $\mathrm{p}<0.05$ ) (Table 1).

Comparing tracheal aspirates with sputum samples that showed Candida spp. growth, statistically significant differences were observed between patients aged less or more than 60 years old, between C. albicans and non-albicans, as well as between the number of identified yeast species (Fisher test; $\mathrm{p}<0.05$ ) (Table 2). The chance of identifying Candida spp. is higher in patients 


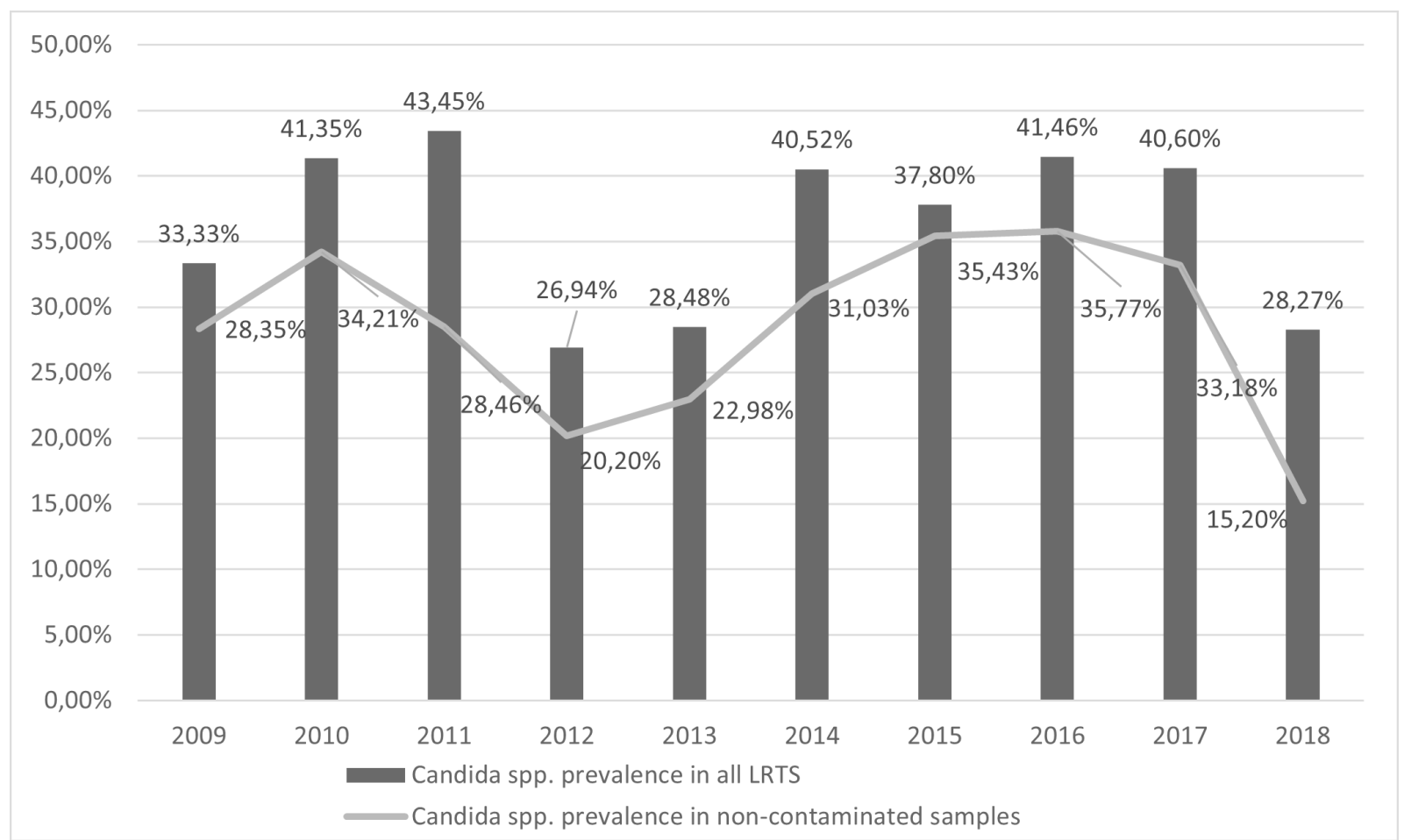

Fig. 2. Candida spp. in the total number of lower respiratory tract samples compared to the non-contaminated samples

Table 1. Comparison of different Candida spp. in LRTS

\begin{tabular}{lccc} 
& C. albicans & C. non-albicans & C. albicans and C. non-albicans \\
Number of samples & 445 & 357 & 70 \\
Mean & 61.51 & 65.76 & 64.42 \\
Standard Deviation & 16.293 & 13.744 & 15.63 \\
Median & 64 & 69 & 69 \\
Min & 6 & 21 & 12 \\
Max & 94 & 99 & 88 \\
Normal distribution & no & no & yes \\
\hline
\end{tabular}

Table 2. Candida spp. - positive tracheal aspirate and sputum samples; $\boldsymbol{n}$ represents the number of analysed data after exclusion of incomplete data; $\mathrm{Cl}$ (confidence interval); $\mathrm{p}$ ( $\mathrm{p}$-value), $\mathrm{n}$ represents the number of analysed data

\begin{tabular}{|c|c|c|c|c|c|c|}
\hline & & $\mathbf{n}$ & Tracheal aspirate & Sputum samples & p & OR \\
\hline \multirow[t]{2}{*}{ Age } & $>60$ years old & \multirow{2}{*}{544} & 322 (32.07\%) & 317 (31.57\%) & \multirow{2}{*}{0.0001} & 1.671 \\
\hline & $<=60$ years old & & $138(13.75 \%)$ & 227 (22.61\%) & & (Cl 1.285-2.172) \\
\hline \multirow[t]{2}{*}{ Gender } & Female & \multirow{2}{*}{893} & $182(16.01 \%)$ & 195 (17.15\%) & \multirow{2}{*}{0.3122} & 1.141 \\
\hline & Male & & $342(30.08 \%)$ & $418(36.76 \%)$ & & $(\mathrm{Cl} 0.8906-1.461)$ \\
\hline \multirow[t]{2}{*}{ Candida spp. } & C. albicans & \multirow{2}{*}{470} & 224 (24.27\%) & 281 (30.44\%) & \multirow{2}{*}{0.0019} & 0.6579 \\
\hline & C. non-albicans & & $229(24.81 \%)$ & $189(20.48 \%)$ & & (Cl 0.5069-0.8538) \\
\hline \multirow{2}{*}{$\begin{array}{l}\text { The number of } \\
\text { Candida spp. }\end{array}$} & One species & \multirow{2}{*}{589} & $453(39.56 \%)$ & $470(41.05 \%)$ & \multirow{2}{*}{0.0002} & 1.779 \\
\hline & Two and more species & & $78(6.81 \%)$ & $144(12.58 \%)$ & & (Cl 1.312-2.412) \\
\hline
\end{tabular}

aged over 60 years old, especially from tracheal aspirates. C. albicans is more prevalent in sputum samples.

Comparing tracheal aspirates with sputum samples that showed Candida spp. growth, statistically significant differences were observed between patients aged $<=$ or $>$ than 60 years old, between $C$. albicans and $C$. non-albicans, as well as between the number of identified yeast species (Fisher test; $\mathrm{p}<0.05$ ) (Table 2). Candida spp. are more prevalent in male patients, and are more often identified in sputum samples. The chances of identifying one species are greater in both sputum and tracheal aspirates. 
Table 3. Contaminated and non-contaminated sputum samples that showed Candida growth; $\mathrm{n}$ represents the number of analysed data after exclusion of incomplete data; OR (odds ratio); $\mathrm{Cl}$ (confidence interval); $\mathrm{p}$ ( $p$-value)

\begin{tabular}{|c|c|c|c|c|c|c|}
\hline & \multirow{2}{*}{\multicolumn{2}{|c|}{ Parameter }} & \multicolumn{2}{|c|}{ Frequency and Percentage } & \multirow[b]{2}{*}{$\mathbf{p}$} & \multirow[b]{2}{*}{ OR } \\
\hline & & & $\begin{array}{c}\text { Contaminated sputum } \\
\text { samples }\end{array}$ & $\begin{array}{l}\text { Non-contaminated } \\
\text { sputum samples }\end{array}$ & & \\
\hline \multirow[t]{5}{*}{ Age } & \multirow[t]{5}{*}{$n=544$} & $\begin{array}{l}>40 \text { years old } \\
<=40 \text { years old }\end{array}$ & $\begin{array}{c}197(36.21 \%) \\
35(6.43 \%)\end{array}$ & $\begin{array}{c}286(52.57 \%) \\
26(4.78 \%)\end{array}$ & 0.0189 & $\begin{array}{c}1.954 \\
(\mathrm{Cl} 1.140-3.35)\end{array}$ \\
\hline & & $>60$ years old & $126(23.16 \%)$ & $191(35.11 \%)$ & \multirow{2}{*}{0.114} & \multirow{2}{*}{$\begin{array}{c}0.753 \\
(\mathrm{Cl} 0.5336-1.063)\end{array}$} \\
\hline & & $<=60$ years old & $106(19.49 \%)$ & $121(22.24 \%)$ & & \\
\hline & & $>80$ years old & $12(2.21 \%)$ & $26(4.78 \%)$ & \multirow{2}{*}{0.175} & \multirow{2}{*}{$\begin{array}{c}1.667(\mathrm{Cl} 0.822- \\
3.378)\end{array}$} \\
\hline & & $<=80$ years old & $220(40.44 \%)$ & $286(52.57 \%)$ & & \\
\hline \multirow[t]{2}{*}{ Gender } & \multirow[t]{2}{*}{$n=612$} & Female & $86(14.05 \%)$ & $109(17.81 \%)$ & \multirow{2}{*}{0.5389} & \multirow{2}{*}{$\begin{array}{c}1.124 \\
(\mathrm{Cl} 0.7971-1.584)\end{array}$} \\
\hline & & Male & $172(28.10 \%)$ & 245 (40.03\%) & & \\
\hline \multirow[t]{2}{*}{ Candida spp. } & \multirow[t]{2}{*}{$n=470$} & C. albicans & $122(25.96 \%)$ & $159(33.83 \%)$ & \multirow{2}{*}{0.0004} & \multirow{2}{*}{$\begin{array}{c}0.5048 \\
(\mathrm{Cl} 0.3469-0.7345)\end{array}$} \\
\hline & & C. non-albicans & $114(24.26 \%)$ & $75(15.96 \%)$ & & \\
\hline \multirow{2}{*}{$\begin{array}{l}\text { The number of } \\
\text { Candida spp. }\end{array}$} & \multirow[t]{2}{*}{$n=589$} & One species & $197(33.45 \%)$ & $273(46.35 \%)$ & \multirow[b]{2}{*}{0.0358} & \multirow{2}{*}{$\begin{array}{c}1.1599 \\
(\mathrm{Cl} 1.041-2.457)\end{array}$} \\
\hline & & $\begin{array}{l}\text { Two and more } \\
\text { species }\end{array}$ & $37(6.28 \%)$ & $82(13.92 \%)$ & & \\
\hline
\end{tabular}

Patients aged more than 40 years old were more prone to provide contaminated sputum specimens (Fisher test; $\mathrm{p}<0.05$ ) (Table 3). No statistical significance was observed between patients aged less than or equal to or greater than 60 years old or between patients aged less than 80 or equal to or greater than 80 years old (Fisher test; $\mathrm{p}>0.05$ ).

Comparing the contaminated sputum samples, with the sputum samples considered non-contaminated, statistically significant differences were observed between the diagnosis of $C$. albicans and non-albicans species, as well as in the number of identified isolates (Fisher test; $\mathrm{p}<0.05$ ) (see Table 3).

Significantly, more albicans species were found in non-contaminated sputum samples, mostly as single species.

\section{DISCUSSION}

Pneumonia with Candida spp. is considered a secondary effect: usually an immunocompromised patient has a primary infection site (e.g. the skin, the gastrointestinal tract) from which Candida cells disseminate through the bloodstream and infect the lungs. Because the fungal serology often remains negative, these cases are seldom considered, and the definitive diagnosis is made post-mortem, in the pathology department [12]. However, in a two-year study conducted in an adult ICU, no single case of confirmed Candida pneumonia was reported [13].
Despite the frequent identification of Candida spp. in respiratory tract secretions, pneumonia with Candida spp. is rare, and positive cultures are most probably due to colonisation $[13,14]$. Should these results be reported? If yes, how should they be interpreted? Barenfanger and al. (2003) showed that over $70 \%$ of the clinicians do not consider Candida spp. in respiratory tract secretions and rarely initiate antifungal therapy [15]. The mortality in patients with positive cultures with Candida spp. was not influenced by antifungal therapy, so some researchers consider that a limited identification can decrease the length of stay in the hospital, reduce costs, and limit the unnecessary usage of antifungal agents $[15,16]$. On the one hand, higher antifungal usage is associated with increased resistance rates [17], supporting the necessity of limiting unnecessary antifungal treatment. On the other hand, the state of colonisation with Candida spp. is associated with prolonged hospitalisation in ICU and increased costs, probably because of the toxic effect of antifungal drugs [18].

Over half of the samples included in the current study came from the hospital's ICU, from patients with impaired immune systems that are, theoretically, prone to Candida spp. pneumonia. The rarity of such reported cases in the literature suggests that a positive LRTS sample indicates the state of colonisation with Candida spp. Although the respiratory tract was considered aseptic in the past, the bronchial tree has a characteristic microbiota that plays a role in the development of lung pathology [19]. The respiratory tract's mycobiota is still highly 
enigmatic, so the yeast cells come, most probably, from the oral cavity [20]. The high prevalence of Candida spp. in LRTS raises questions about the collection methods used for collecting such samples, and positive results are difficult to interpret from a clinical perspective.

In the current study, even after excluding the samples considered contaminated, according to the Bartlett score, the prevalence of Candida spp. in respiratory tract secretions was high. How effective are the protocols used to assess sputum quality? Candida spp. associated pneumonia is extremely rare. Nevertheless, in the current study, the number of Candida spp. isolates was high, in spite of all contaminated samples being excluded.

Multiple criteria can be used in a laboratory for judging the quality of sputum samples [21-23], with different reproducibility rates [2]. An accurate and reliable microbiological examination is more than desirable. Although initially it was thought that sputum Gram stain could not provide enough information in the absence of culturing the sample, this might not be entirely true. Gram staining can play a key role in diagnosing pneumonia, and the Bartlett score can predict true lower respiratory tract infections. However, regarding whether samples are contaminated or not, there is only a moderate agreement when the results of Gram stain and sputum sample-culturing are compared [24].

In a study conducted by Gunasekaran et al. (2019), $40 \%$ of the analysed sputum samples were of good quality, as assessed by the Bartlett score [24]. In a study by Fukuyama et al. (2014), the percentage of good quality samples was $71.3 \%$. However, the sputum samples in this study were not obtained exclusively by expectoration, but also by nasotracheal suction. In some centres, clinicians are trained in microbial examination. They can perform a Gram stain in their routine clinical practice, which raises the number of good quality samples sent to the laboratory [25]. In our study, a comparable percentage (68.4\%) of the sputum samples was of good quality and shown to be non-contaminated, although clinicians are not trained in microscopical screening methods in Romania.

Compared with bacterial isolates, the presence of Candida spp. in respiratory tract secretions, might be associated with systemic inflammation, and it might imply a poorer clinical outcome [18]. This may be due to the B-glucan, a fungal cell wall component that can activate the immune response [21]. However, in a prospective observational study on 598 patients, Wil- liamson et al. (2011) contradict the association of inflammation with the presence of Candida spp. in respiratory tract cultures. Patients with positive cultures for Candida spp. had similar inflammation levels as the control group, but their clinical evolution was worse [18]. Terraneo et al. (2016) concluded that even if Candida spp. in RTS is associated with the initial severity of the cases, its identification does not influence the outcomes, regardless of the antifungal usage [22]. The association between Candida colonisation and inflammation and its impact on the medical cases' clinical evolution remains highly controversial.

The mycological examination of LRTS can help predict the risk of developing severe Candida infections as a Colonization Index (CI) (number of positive sites / number of tested sites) greater than 0.5 indicates an increased risk of deep-seated fungal infections and may justify antifungal therapy. Blood cultures are never taken into consideration while calculating the CI [23, 26, 27].

It is well known that cross-kingdom relationships between bacteria and fungi can impact the host metabolism and immunity, hence playing a role in the onset and development of diseases [28]. For patients admitted to ICU, Candida spp. airway colonisation might increase the risk of developing ventilator-associated pneumonia (VAP) with multi-drug resistant bacteria. It might even worsen the evolution of VAP regardless of the primary causative agent [29]. For example, Candida spp. is often isolated next to Pseudomonas aeruginosa. A study on mice showed that C. albicans inhibited reactive oxygen species (ROS) production in alveolar macrophages and the rats that were colonised with C. albicans had an increased risk of developing VAP with P. aeruginosa [30]. In mixed biofilms, the interspecies competition between $C$. albicans and $P$. aeruginosa results in increased virulence factors and an enhanced mutability [31]. However, the relationship between $C$. albicans and $P$. aeruginosa is a complicated one: in an in vitro dual environment, they mutually suppress a biofilm development, which can be defined as antagonism. In vivo, either species can alter colonisation by the other, favouring or inhibiting disease [32, 33]. Some authors consider the usage of antifungals for reducing the risk of VAP in patients colonised with Candida spp. [34]. However, such studies have severe limitations, and clinical trials are needed to become a standard of practice. 
In a prospective study on 3648 patients with candidemia, Pfaller et al. (2012) [35] the 90-day survival rate was $61.3 \%$, candidemia occurring mainly in immunosuppressed hosts, i.e. patients with concurrent bacterial infections, corticosteroid therapy, mechanical ventilation, diabetes, and patients that underwent non-transplant related surgeries during hospitalisation. Whether the source of the infection is endogenous or exogenous is debated in the literature. Cross-contamination between colonised and un-colonised patients is not documented, so invasive Candida spp. has an endogenous start point. Calculating the $\mathrm{CI}$ can help practitioners detect patients at high risk for developing candidemia [27]. In studies of autopsy-proved invasive Candida spp., antemortem blood cultures were positive in $21-71 \%$ of the cases. Nonculture diagnostic methods are not ready to be implemented into the current standard of practice, and blood cultures are an excellent diagnostic method for invasive infections, at the moment [36]. In this context, calculating the CI might have clinical value, especially for patients with impaired immune systems.

The risk of developing Candida infections increases with the number of underlying comorbidities like HIV infection, malignancies diabetes mellitus, chronic obstructive pulmonary disease, chronic liver disease, and severe heart failure. Also, the previous usage of antifungal and/or antibiotic drugs, corticosteroids, undergoing surgical procedures, parenteral nutrition, dialysis, mechanical ventilation, or septic shock favour the onset of infections [37]. The oral colonization with Candida spp. in diabetic patients may play a role in how the immune system will later respond to glucose intake [38]. Next to candidiasis, oral diseases are the most common infections in diabetic patients [39] and diabetic patients with poor glucoregulation are more often carriers of Candida spp. [40].

The present study emphasises the improper collection of sputum, as the number of saliva samples sent to the laboratory is still high. Although sputum should be sterile in the lack of infections, in reality, the collection of sputum through expectoration permits microorganisms, living in the oral cavity, to contaminate LRTS. Patients admitted to an ICU are often intubated, and around $45 \%$ of the intubated patients became colonised with Candida spp. after 48 hours [41]. It is accepted that prolonged ICU hospitalisations, the number of invasive manoeuvres a patient is receiving, bacterial sepsis, and exposure to broad-spectrum antibiotics are risk factors for Candida spp. colonisation [42].
The oral mycobiome composition has received scant attention, mainly because many fungi are difficult to culture. Although the composition and, most importantly, the oral mycobiome's role is a work in progress, Candida spp. are the most studied and generally considered the most important of the mycrobiome. The oral mycobiome changes with time, and it is influenced by factors such as the salivary flow rate, the Body Mass Index, and geographical background [32]. To colonise the oral cavity, the yeast cells have to first attach to epithelial cells. Proteins like als 1-7, als 9, eap1, hwp1, pga 1 mediate the attachment process, thus playing a pivotal role in colonising a host niche. To increase the survival chances, Candida cells can form biofilms, complex heterogeneous structures from which the fungal cells can detach and generate novel communities in other body niches [43].

Endotracheal tubes become contaminated with oral cavity flora within twenty-four hours of intubation, and a longer incubation time increased the proliferation rate of microorganisms on such devices [44]. As proven in vitro, Candida spp. can form biofilms in a short timeframe: in approximately eleven hours microcolonies can be observed. The intermediate phage of biofilm formation takes 12-30 hours, and the maturation stage takes approximately 38-72 hours [45]. In vivo, biofilm formation starts even sooner, within eight hours [46]. After the adhesion to a substrate, the fungal cells proliferate and are further engulfed in an extracellular matrix. As opposed to planktonic cells (free-floating cells), sessile cells (part of the biofilm) are more resistant to various host defence mechanisms, either by altering the host's immune response or by exhibiting epigenetic resistance to antimicrobials. The cells released from a preformed biofilm have an increased virulence $[43,47$, 48]. If neutrophils release NETs (extracellular traps) in response to planktonic Candida cells, the extracellular matrix impairs NET production [49]. C. albicans cells dispersed from biofilms are developmentally distinct compared to planktonic cells: they have enhanced virulence-associated characteristics like adherence and filamentation. They are transcriptionally reprogramed to acquire nutrients and metabolise alternative carbon sources $[50,51]$.

As aging is associated with changes in the oral cavity's ecology, due to medications, denture wearing, or a deteriorated general health status, the increased number of LRTS samples collected from patients aged over 60 years old meets the expectations. The oral microbi- 
ome of the elderly consists of a high level of staphylococci, Candida spp., lactobacilli, and actinomycetes [3].

\section{CONCLUSIONS}

The sample collection methods play a crucial role in ensuring that patients are correctly diagnosed and provided with the appropriate treatment. Given the high percentage of contaminated sputum samples, the study results emphasise the significance and need for highquality procedures when collecting specimens. Furthermore, the results indicated the feasibility and benefits of microscope screening methods in respiratory tract secretions' routine workouts. Every clinical case should be judged on its own merits.

\section{AUTHOR CONTRIBUTIONS}

Conceptualization, C.N.C., A.D.M., F.T., A.M.; methodology, C.N.C., A.D.M., A.M.; software, C.N.C., A.D.M., A.M; validation, C.N.C., A.S., A.D.M., A.M., B.K, F.T., C.V., I.P., formal analysis, C.N.C., A.S., A.D.M., A.M., B.K; investigation, C.N.C, B.K., C.V., I.P.; resources, C.N.C., A.D.M., A.M., F.T.; data curation, C.N.C, B.K., C.V., I.P..; writing-original draft preparation, C.N.C., A.D.M., A.M.; writing- C.N.C., A.S., A.M.; visualization, C.N.C., A.S., A.D.M., A.M. B.K, F.T., C.V., I.P.; supervision, A.M.; project administration, CNC, A.M.; funding acquisition, C.N.C. All authors have read and agreed to the published version of the manuscript.

\section{FUNDING}

This research was funded by the University of Medicine, Pharmacy, Science and Technology "George Emil Palade" of Târgu Mureș Research Grant number 10127/2/17.12.2020.

\section{- CONFLICTS OF INTEREST}

The authors declare no conflict of interest.

\section{ETHICAL APPROVAL}

The ethical approval was waived by the Ethics Committee of Mures County Clinical Hospital, Decision no 15596/19.10.2018 in view of the retrospective nature of the study and all the procedures being performed were part of the routine care.

\section{DATA}

Data available on request from the authors.

\section{REFERENCES}

1. Leroy O, Gangneux J-P, Montravers P, et al. Epidemiology, management, and risk factors for death of invasive Candida infections in critical care: A multicenter, prospective, observational study in France (2005-2006). Critical Care Medicine 2009; 37: 1612-1618.

2. Wong LK, Barry AL, Horgan SM. Comparison of six different criteria for judging the acceptability of sputum specimens. Journal of Clinical Microbiology 1982; 16: 627-631.

3. Belibasakis GN. Microbiological changes of the ageing oral cavity. Archives of Oral Biology 2018; 96: 230-232.

4. Fanello S, Bouchara JP, Sauteron M, et al. Predictive value of oral colonization by Candida yeasts for the onset of a nosocomial infection in elderly hospitalized patients. Journal of Medical Microbiology, 2006; 55: 223-228.

5. Ayhan M, Birengel MS, Yilmaz G, et al. Evaluation of Risk Factors for Candida Colonization and Infection in Non-Neutropenic Intensive Care Patients. Turkiye Klinikleri J Med Sci 2019; 39: 251-257.

6. Olaechea PM, Palomar M, León-Gil C, et al. Economic Impact of Candida Colonization and Candida Infection in the Critically III Patient. Eur J Clin Microbiol Infect Dis 2004; 23: 323-330.

7. Wisplinghoff $H$, Bischoff $T$, Tallent SM, et al. Nosocomial Bloodstream Infections in US Hospitals: Analysis of 24,179 Cases from a Prospective Nationwide Surveillance Study. Clin Infect Dis 2004; 39: 309-317.

8. Barchiesi F, Orsetti E, Mazzanti S, et al. Candidemia in the elderly: What does it change? PLOS ONE 2017; 12: e0176576.

9. Antinori S, Milazzo L, Sollima S, et al. Candidemia and invasive candidiasis in adults: A narrative review. European Journal of Internal Medicine 2016; 34: 21-28.

10. Sadeghi G, Ebrahimi-Rad M, Mousavi SF, et al. Emergence of non-Candida albicans species: Epidemiology, phylogeny and fluconazole susceptibility profile. Journal de Mycologie Médicale 2018; 28: 51-58.

11. Lamoth F, Lockhart SR, Berkow EL, et al. Changes in the epidemiological landscape of invasive candidiasis. J Antimicrob Chemother 2018; 73: i4-i13.

12. Isolated Candida infection of the lung. Respiratory Medicine Case Reports 2015; 16: 18-19.

13. Meersseman W, Lagrou K, Spriet I, et al. Significance of the isolation of Candida species from airway samples in critically ill patients: a prospective, autopsy study. Intensive Care Med 2009; 35: 1526-1531.

14. Sousa AS, Ferrito C, Paiva JA. Intubation-associated pneumonia: An integrative review. Intensive and Critical Care Nursing 2018; 44: 45-52. 
Available online at: www.jccm.ro

15. Barenfanger J, Arakere P, Cruz RD, et al. Improved Outcomes Associated with Limiting Identification of Candida spp. in Respiratory Secretions. Journal of Clinical Microbiology 2003; 41: 5645-5649.

16. Pendleton KM, Huffnagle GB, Dickson RP. The significance of Candida in the human respiratory tract: our evolving understanding. Pathog Dis; 75. Epub ahead of print 1 April 2017. DOI: $10.1093 /$ femspd/ftx029.

17. Castanheira M. Fungemia Surveillance in Denmark Demonstrates Emergence of Non-albicans Candida Species and Higher Antifungal Usage and Resistance Rates than in Other Nations. J Clin Microbiol 2018; 56: e01907-17.

18. Williamson DR, Albert M, Perreault MM, et al. The relationship between Candida species cultured from the respiratory tract and systemic inflammation in critically ill patients with ventilator-associated pneumonia. Can J Anaesth 2011; 58: 275-284.

19. Hilty M, Burke C, Pedro $H$, et al. Disordered Microbial Communities in Asthmatic Airways. PLOS ONE 2010; 5: e8578.

20. Zhang $D$, Wang $Y$, Shen $S$, et al. The mycobiota of the human body: a spark can start a prairie fire. Gut Microbes 2020; 11: 655-679.

21. Young S-H, Ostroff GR, Zeidler-Erdely PC, et al. A Comparison of the Pulmonary Inflammatory Potential of Different Components of Yeast Cell Wall. Journal of Toxicology and Environmental Health, Part A 2007; 70: 1116-1124.

22. Terraneo S, Ferrer M, Martín-Loeches I, et al. Impact of Candida spp. isolation in the respiratory tract in patients with intensive care unit-acquired pneumonia. Clinical Microbiology and Infection 2016; 22: 94.e1-94.e8.

23. Sanguinetti M, Posteraro B, Lass-Flörl C. Antifungal drug resistance among Candida species: mechanisms and clinical impact. Mycoses 2015; 58 Suppl 2: 2-13.

24. Gunasekaran J, Saksena R, Jain M, et al. Can sputum gram stain be used to predict lower respiratory tract infection and guide empiric antimicrobial treatment: Experience from a tertiary care hospital. Journal of Microbiological Methods 2019; 166: 105731.

25. Fukuyama H, Yamashiro S, Kinjo K, et al. Validation of sputum Gram stain for treatment of community-acquired pneumonia and healthcare-associated pneumonia: a prospective observational study. BMC Infect Dis 2014; 14: 534.

26. Azoulay E, Cohen Y, Zahar J-R, et al. Practices in non-neutropenic ICU patients with Candida-positive airway specimens. Intensive Care Med 2004; 30: 1384-1389.

27. Eggimann P, Pittet D. Candida colonization index and subsequent infection in critically ill surgical patients: 20 years later. Intensive Care Med 2014; 40: 1429-1448.

28. Nguyen LDN, Viscogliosi E, Delhaes L. The lung mycobiome: an emerging field of the human respiratory microbiome. Front Microbiol 2015; 6: 89.

29. Hamet M, Pavon A, Dalle F, et al. Candida spp. airway colonization could promote antibiotic-resistant bacteria
The Journal of Critical Care Medicine 2021;7(3) • 225

selection in patients with suspected ventilator-associated pneumonia. Intensive Care Med 2012; 38: 1272-1279.

30. Roux D, Gaudry S, Dreyfuss D, et al. Candida albicans impairs macrophage function and facilitates Pseudomonas aeruginosa pneumonia in rat*. Critical Care Medicine 2009; 37: 10621067.

31. Trejo-Hernández A, Andrade-Domínguez A, Hernández M, et al. Interspecies competition triggers virulence and mutability in Candida albicans - Pseudomonas aeruginosa mixed biofilms. The ISME Journal 2014; 8: 1974-1988.

32. Bandara HMHN, Panduwawala CP, Samaranayake LP. Biodiversity of the human oral mycobiome in health and disease. Oral Diseases 2019; 25: 363-371.

33. Fourie R, Pohl CH. Beyond Antagonism: The Interaction Between Candida Species and Pseudomonas aeruginosa. Journal of Fungi 2019; 5: 34.

34. Nseir S, Jozefowicz E, Cavestri B, et al. Impact of antifungal treatment on Candida-Pseudomonas interaction: a preliminary retrospective case-control study. Intensive Care Med 2007; 33 : 137-142.

35. Pfaller M, Neofytos D, Diekema D, et al. Epidemiology and outcomes of candidemia in 3648 patients: data from the Prospective Antifungal Therapy (PATH Alliance ${ }^{\circledR}$ ) registry, 20042008. Diagnostic Microbiology and Infectious Disease 2012; 74: 323-331.

36. Clancy CJ, Nguyen MH. Finding the "Missing 50\%" of Invasive Candidiasis: How Nonculture Diagnostics Will Improve Understanding of Disease Spectrum and Transform Patient Care. Clin Infect Dis 2013; 56: 1284-1292.

37. Fortún J, Martín-Dávila P, Gómez-García de la Pedrosa E, et al. Emerging trends in candidemia: A higher incidence but a similar outcome. Journal of Infection 2012; 65: 64-70.

38. Adriana Monea, Luigi Santacroce, Massimo Marrelli, et al. Oral candidiasis and inflammatory response\&58; A potential synergic contribution to the onset of Type-2 Diabetes Mellitus. Australasian Medical Journal 2017; 10: 550-556.

39. Rodrigues CF, Rodrigues ME, Henriques M. Candida sp. Infections in Patients with Diabetes Mellitus. Journal of Clinical Medicine 2019; 8: 76.

40. Petrovic SM, Radunovic M, Barac M, et al. Subgingival areas as potential reservoirs of different Candida spp in type 2 diabetes patients and healthy subjects. PLOS ONE 2019; 14: e0210527.

41. Ferreira EG, Yatsuda F, Pini M, et al. Implications of the presence of yeasts in tracheobronchial secretions of critically ill intubated patients. EXCLI J 2019; 18: 801-811.

42. Ergin F, Eren Tülek N, Yetkin MA, et al. Evaluation of Candida colonization in intensive care unit patients and the use of Candida colonization index. Mikrobiyol Bul 2013; 47: 305-317.

43. Ciurea CN, Kosovski I-B, Mare AD, et al. Candida and Candidiasis-Opportunism Versus Pathogenicity: A Review of the Virulence Traits. Microorganisms 2020; 8: 857.

44. Perkins SD, Woeltje KF, Angenent LT. Endotracheal tube 
226 The Journal of Critical Care Medicine 2021;7(3)

biofilm inoculation of oral flora and subsequent colonization of opportunistic pathogens. International Journal of Medical Microbiology 2010; 300: 503-511.

45. Cavalheiro M, Teixeira MC. Candida Biofilms: Threats, Challenges, and Promising Strategies. Front Med 2018; 5: 28.

46. Andes D, Nett J, Oschel P, et al. Development and Characterization of an In Vivo Central Venous Catheter Candida albicans Biofilm Model. Infection and Immunity 2004; 72: 6023-6031.

47. Eix EF, Nett JE. How Biofilm Growth Affects Candida-Host Interactions. Front Microbiol 2020; 11: 1437.

48. Finkel JS, Mitchell AP. Genetic control of Candida albicans
Available online at: www.jccm.ro

biofilm development. Nature Reviews Microbiology 2011; 9: 109-118.

49. Johnson CJ, Cabezas-Olcoz J, Kernien JF, et al. The Extracellular Matrix of Candida albicans Biofilms Impairs Formation of Neutrophil Extracellular Traps. PLOS Pathogens 2016; 12: e1005884.

50. Uppuluri P, Chaturvedi AK, Srinivasan A, et al. Dispersion as an Important Step in the Candida albicans Biofilm Developmental Cycle. PLoS Pathog 2010; 6: e1000828.

51. Wall G, Montelongo-Jauregui D, Bonifacio BV, et al. Candida albicans biofilm growth and dispersal: contributions to pathogenesis. Current Opinion in Microbiology 2019; 52: 1-6. 